\title{
CÀI ĐặT CÁC THUẬT TOÁN BẢO ĐẢM TÍNH NHẤT QUÁN DŨ LIỆU CHO MÔ HÌNH CƠ SỞ DŨ LIỆ QUAN HỆ
}

\author{
Phạm Quang Trung \& NGUYẼN XUÂN HUY
}

Viện Công nghệ Thông tin

\begin{abstract}
Summary. Data integrity preservation is an important ruequirement in designing databases satisfied to build normal forms of relational schemes. All algorithms that translate a form of schema into a normal form require to define keys of the schema. Therefore a key finding problem is very important for designing database. There have been many solutions for this problem. In this paper, we present a solution that base on the concept of translation of relational schemes, which translates the prime schema into the class of schemes that made key finding problem simpler. Basis factors of relational schema are an amount of attributes, of functional dpendencies. That has concern with a completcity of closure, key, and cover finding problem... The purpose of the translation to simplify the prime schema by rejecting some attributes, which are not belong to or are belong to all keys of prime schema is the result after adding with a set of attributes belong DABASCHE that is a solution of implementation of that algorithm. We present some important functions of the module DABASCHE for designing relational databases and it's basic data structures in the language that simulates the Pascal programming language.
\end{abstract}

\section{Mờ ĐẦU}

Việc đảm bảo tính nhất quán cưa dữ liệu là một yêu cầu quan trọng trong thiết kế cơ sở dữ liệu, đáp úng yêu cầu xây dựng các lược đồ quan hệ (LDQH) ở dạng chuẩn. Hầu hết các thuật toán đưa $L Đ Q H$ về dạng chuẫn đều đòi hởi phải xác định khoá của lược đồ. Do đó bài toán tìm tập hợp các khoá cưa một $L Đ Q H$ để có thể xác định khoá chỉ định tối ưu nhất là có ý nghiã. Đã có nhiều cách giải bài toán tìm khoá. Phương pháp biến đổi một $L Đ Q H$ đưa về lóp lược đồ có cách giải bài toán tìm khoá đơn giản hơn được trình bày trong bài này. Những yếu tố cơ bản cưa $L D Q H$ liên quan đến độ phức tạp của việc xác định bao đóng, tìm khoá, phủ tối thiểu ... là số các thuộc tính, và số lượng các phụ thuộc hàm. Với ý tưởng làm đơn giản hoá lược đồ ban đầu bằng cách loại bớt các thuộc tính không mang thông tin về khoá và cá các thuộc tính nằm trong mọi khoá, việc xác định các tập hợp khoá của lược đồ nhận được sẽ thuận lợi hơn, và với kết quả lý thuyết đã được chưng minh, tập khoá của LDQH ban đầu chính là kết quá của phép phối hợp tập khoá đã nhận được với tập giao của các lược đồ ban đầu. Cùng với các cơ sớ lý thuyết của phương hpáp này, bài báo trình bày một giải pháp cài đặt nhằm cung cấp công cụ cho nhũng người thiết kế cơ sở dữ liệu vận dụng phép biến đổi 
lược đồ quan hệ để xác định tập hợp khóa của LDQH, đó là module DABASCHE, bao gồm giới thiệu các chức năng chính và thông qua ngôn ngữ tựa Pascal thể hiện các cấu trúc dữ liệu cơ bản của module DABASCHE.

Cho $U=\left\{a_{1}, \ldots, a_{n}\right\}$ là một tập hũu hạn khác trống, các ký hiệu $a_{i}, i=1, \ldots, n$, gọi là các thuộc tính. Với mỗi $i$, tập khác trống $\operatorname{dom}\left(a_{i}\right)$ gọi là miền xác định cưa $a_{i}$. Kí hiệu tập hội với mọi $i: D=\cup_{i=1}^{n} \operatorname{dom}\left(a_{i}\right)$. Một quan hệ $R$ trên tập thuộc tính $U$ là tập hữu hạn các ánh xạ $\left\{r_{1}, \ldots, r_{n}\right\}$ từ tập $U$ vào tập $D$ sao cho với mọi $a_{i} \in U$ ta luân có: $r\left(a_{i}\right) \in \operatorname{dom}\left(a_{i}\right)$.

Các ánh xạ $r_{i} \in R$ được gọi là các bộ (tuples) của quan hệ $R$.

Các ký hiệu. Theo truyền thống trỉnh bày cứa lý thuyết quan hệ, trong bài này sử dụng các ký hiệu sau:

- Quan hệ $R$ trên tập thuộc tính $U$ được kí hiệu là $R(U)$.

- Hợp của hai tập thuộc tính $X, Y$ sẽ được viết là $X Y$.

- Vói quan hệ $R$ trên tập thuộc tính $U, r$ là một bộ cưa $R, X$ là tập con của $U$ thì giá trị của $r$ trên $X$ được viết là $r(X)$.

Cho tập hợp $U$ và $M, N$ là họ các tập con của $U$, tập $Z \subseteq U$. Ta định nghiã phép toán $\oplus$ như sau:

$$
\begin{aligned}
Z \oplus N & =\{Z Y \mid Y \in N\}, \\
M \oplus N & =\{X Y \mid X \in M, Y \in N\} .
\end{aligned}
$$

Cho $R(U)$ và hai tập thuộc tính $X, Y \subseteq U$. Ta nói rằng tập thuộc tính $Y$ phụ thuộc hàm vào tập thuộc tính $X$ và viết $X \rightarrow Y$ nếu: với mọi bộ $r_{1}, r_{2}$ trong quan hệ $R$ từ: $r_{1} \cdot X=r_{2} . X \Rightarrow r_{1} \cdot Y=r_{2} . Y$.

Cho $F$ là tập các phụ thuộc hàm đã biết trên quan hệ $R$, và cho $X \rightarrow Y$ là một phụ thuộc hàm. Ta nói $X \rightarrow Y$ là suy diễn được từ $F$ nếu vói mỗi quan hệ $R(U)$ thoá các phụ thuộc hàm của $F$ thì cũng thỏa phụ thuộc hàm $X \rightarrow Y$, kí hiệu $F \Rightarrow X \rightarrow Y$.

Dịnh nghiã. $F^{+}$là bao đóng cưa tập phụ thuộc hàm là tập chứa tất cá phụ thuộc hàm có thể suy diễn được từ $F: F^{+}=\{X \rightarrow Y \mid F \Rightarrow X \rightarrow Y\}$.

Lược đồ quan hệ $\omega=(U, F)$ là một cặp trong đó $U$ là một tập hũu hạn các thuộc tính và $F$ là tập hữu hạn các phụ thuộc hàm trên $U$. Cho lược đồ quan hệ (LĐQH) $\omega=(U, F)$ và tập $X \subseteq U$. Bao đóng của tập $X$ dưới $\omega$, kí hiệu $X_{\omega}^{+}$là tập lón nhất các thuộc tính $Y \subseteq U$ sao cho: $F: \Rightarrow X \rightarrow Y$. (Kí hiệu $X^{+}$được sứ dụng nếu không có sự nhầm lẫn).

Việc kiểm chứng $F \Rightarrow X \rightarrow Y$ được đám bảo qua bồ đề sau đây:

Bổ đề. $F \Rightarrow X \rightarrow Y \Longleftrightarrow Y \subseteq X^{+}$.

Cho lược đồ quan hệ $\omega=(U, F)$, một tập con $X$ của $U$ là siêu khoá cúa lược đồ $\omega$ nếu $X \rightarrow U \in F^{+}$. Một tập con $K \subseteq U$ là một khoá cưa $\omega$ nếu $K$ là một siêu khoá của $\omega$ và không tồn tại $K^{\prime} \subset K$ nào sao cho $K^{\prime} \rightarrow U \in F^{+}$. 
Thuật toán tìm khoá cớ bản dựa vào ý tưởng xuất phát từ một siêu khoá, tiến hành loại dần các thuộc tính cho đến khi nhận được tập nhơ nhất.

\section{PHÉP BIÊN DỔI LỰ̛̣C Dồ QUAN HỆ}

Cho một $\mathrm{LDQH} \omega=(U, F)$ và cho $Z \subseteq U$, đối với mỗi đối tượng $E$, ( $E$ có thể là tập các thuộc tính, tập các phụ thuộc hàm hoặc chính là lược đồ quan hệ), ta xây dựng đối tượng mới ký hiệu là $E \backslash Z$ bằng cách loại bỏ khói $E$ các phần tử trong $Z$ như sau:

- Trường hợp $E$ là tập thuộc tỉnh, ta định nghiã $E \backslash Z$ là: đới với mỗi phụ thuộc hàm $f: X \rightarrow Y$ trong $E$ thì $f \backslash Z: Z \backslash Z=(U \backslash Z, F \backslash Z)$.

Cho lược đồ quan hệ $\omega=(U, F)$, ký hiệu:

- Tập tất cả các khoá của $\omega$ được ký hiệu là $K_{\omega}$.

- $\forall f \in F$, kí hiệu LEFT(f) và RIGHT(f) là vế trái và vé phải của $f$.

- Định nghiã một số tập hợp như sau:

$I_{\omega}=\bigcap_{k \in \mathcal{K}_{\omega}} K$ tập giao của tát cá các khoá.

$N_{\omega}=U \backslash \underset{k \in K_{\omega}}{\cup} K \quad$ tập các thuộc tính không thuộc vào khoá nào / tập các thuộc tính không nguyên tố).

Dịnh nghiã phép biến dổi LĐQH.

Một lược đồ $\omega^{\prime}=\left(U^{\prime}, F^{\prime}\right)$ là kết quá của phép biến đối LĐQH $\omega=(U, F)$ trên $Z$ nếu $Z \subseteq U$ và $\omega^{\prime}=\omega \backslash Z$ nghiã là $U^{\prime}=U \backslash Z$ và $F^{\prime}=F \backslash Z$.

Sau khi biến đổi, tập phụ thuộc hàm của $\omega^{\prime}$ có thể chứa nhũng phụ thuộc hàm dạng sau đây

$$
\begin{aligned}
& -\Phi \rightarrow \Phi, \\
& -C \rightarrow \Phi \quad(C \subseteq U \backslash Z ; C \# \Phi), \\
& -\Phi \rightarrow C \quad(C \subseteq U \backslash Z ; C \# \Phi) .
\end{aligned}
$$

Theo định nghiã của bao đóng, các phụ thuộc hàm dạng (1) và (2) không bổ xung các phần tử mới trong quá trình suy diễn, do đó về thực chất chúng không được sử dụng trong bất kỳ dãy suy diễn đẽ tìm bao đóng nào, việc bó chúng đi sẽ không làm thay đổi các tập khoá $\mathcal{K}_{\omega^{\prime}}$ của lược đồ $\omega^{\prime}$. Vì vậy, chúng ta ngầm định rằng sau khi thực hiện phép biến đổi trong tập các phụ thuộc hàm cưa lược đồ nhận được sẽ không chứa các phụ thuộc hàm dạng (1) và (2). 
Dịnh lý cơ bản của phép biến đổi. Cho lược đồ quan hệ $\omega=(U, F)$ và cho $Z \subseteq U$. Nếu $\omega^{\prime}=\omega \backslash Z=(U \backslash Z, F \backslash Z)$, thì $\forall X \subseteq U \backslash Z$ ta có: $(Z X)_{\omega}^{+}=Z(X)_{\omega^{\prime}}^{+}$. Dịnh lý. Cho $\omega=(U, F)$ là một lược đồ quan hệ. Ta có $I_{\omega}=U \backslash \underset{f \in F}{\cup}(R I G H T(f) \backslash L E F T(f))$.

Bổ đề. Nếu $M$ là siêu khoá của $\omega$ thì $M \backslash X$ là siêu khoá của $\alpha=\omega \backslash X$; ngực lại nếu $N$ là siêu khoá cứa $\alpha$ thì $N X$ là siêu khoá của $\omega$.

Định lý. Cho $\omega=(U, F)$ là một lược đồ quan hệ và $X$ là tập con cưa $U$. Cho $\alpha=\omega \backslash X$ thì: $+$

$$
\text { 1) } \begin{array}{r}
\text { 1) } \mathcal{K}_{\omega}=\mathcal{K}_{\alpha} \Longleftrightarrow X \subseteq N_{\omega} . \\
\text { 1) } \mathcal{K}_{\omega}=X \oplus \mathcal{K}_{\alpha} \Longleftrightarrow X \subseteq I_{\omega} .
\end{array}
$$

Hệ quả. Cho một lược đồ quan hệ $\omega=(U, F)$ và cho

$$
U^{\prime}=\underset{f \in F}{\cup} R I G H(f) \backslash \bigcup_{f \in F} L E R T(F)
$$

thi

1) $U^{\prime} \subseteq N_{\omega}$.

2) Nếu $X \subseteq I_{\omega} \& Y \subseteq N_{\omega}$ thì $(X Y)_{\omega}^{+} \backslash X \subseteq N_{\omega}$.

Úng dụng phép biến đổi trong việc tìm khoá. Cho lược đồ quan hệ $\omega=(U, F)$, việc tìm khoá của $\omega$ là bài toán NP đầy đủ [8], do đó chúng ta tiến hành bién đổi $\omega$ về một lớp lược đồ đặc biệt mà tập khoá của nó có thể được nhận biết dễ dàng hơn. Phương pháp giải quyết là tìm tập $X$ lớn nhất $\subseteq I_{\omega}$ và tập $Y$ lớn nhất $\subseteq N_{\omega}$, và tính $Z=X\left((X Y)_{\omega}^{+} \backslash X\right)=(X Y)_{\omega}^{+}$, vận dụng phép biến đổi lược đồ quan hệ để chuyển lược đồ $\omega$ thành $\alpha=\omega \backslash Z$. So vói lược đồ $\alpha$ có số lượng thuộc tính ít hơn, và số lượng phụ thuộc hàm cunng có thể ít hơn, do đó việc xác định khoá của $\alpha$ sẽ đơn giản hơn việc xác định khoá của $\omega$. Khi đã có khoá của $\alpha$ ta dễ dàng khôi phục lại được khoá của $\omega$ bằng phép hợp khoá của $\alpha$ với $X$.

Thuật toán chuyển dịch lược đồ quan hệ

Cho lược đồ quan hệ $\omega=(U, F)$, với

$$
\begin{aligned}
& U=\left\{a_{1}, \ldots, a_{n}\right\}, \\
& F=\{L E F T(F) \rightarrow R I G H T(F) \mid f \in F\} .
\end{aligned}
$$

Bước 1. Tìm tập $X$ lón nhất $\subseteq I_{\omega}$, theo định lý đã nêu thì $I_{\omega}$ dễ tính được, nên ta sẽ lấy $X=I_{\omega}=U \backslash \underset{f \in F}{\cup}(R I G H(f) \backslash \operatorname{LEFT}(f))$.

Bước 2. Tìm tập $Y$ lớn nhất $\subseteq N_{\omega}$, theo hệ quả đã nêu thì ta có:

$U^{\prime}=\bigcup_{f \in F}(R I G H(f) \backslash L E F T(f)) \subseteq N_{\omega}$, và do chưa có công thức hiền để tính được $N_{\omega}$ nên ta lấy $Y=U^{\prime}$. 
Bước 3. Tính $Z=X\left((X Y)_{\omega}^{+} \backslash X\right)=(X Y)_{\omega}^{+}$

Bước 4. Tiến hành phép biến đổi lược đồ quan hệ để chuyển lược đồ $\omega$ thành lược đồ $\alpha=\omega \backslash Z$.

Thí dụ. Cho lược đồ quan hệ $\omega=(U, F)$, với $U=a b c d f g h i k$, và

$$
\begin{aligned}
F & =\{a c h \rightarrow b, \\
b h & \rightarrow a c d, \\
a b c i & \rightarrow d k, \\
a d e i & \rightarrow b c g, \\
c g i & \rightarrow a e k, \\
h & \rightarrow b c\},
\end{aligned}
$$

Thực hiện giải thuật nêu trên ta có:

1) Tính tập $X$ :

$$
X=I_{\omega}=U \backslash \cup_{f \in F}(R I G H T(f) \backslash(L E F T(f))=a b c d e g h i k \backslash a b c d e g k=h i .
$$

2) Tinh

$$
Y=U^{\prime}=\cup_{f \in F}(R I G H T(f) \backslash(L E F T(f))=a b c d e g k \backslash a b c d e g h i=k .
$$

3) Tính

$$
\left.Z=X(X Y)_{\omega}^{+} \backslash X\right)=(X Y)_{\omega}^{+}=\left(I_{\omega} U^{\prime}\right)_{\omega}^{+}=(h i k)_{\omega}^{+}=a b c d h i k
$$

4) Lập lược đồ quan hệ chuyển dịch: $\alpha=\omega \backslash Z$

$$
\begin{aligned}
& \alpha=\left(U_{\alpha^{\prime}}, F_{\alpha}\right), \text { với } U_{\alpha}=U \backslash Z=a b c d e g h i k \backslash a b c d h i k=e g, \\
& F_{\alpha}=F \backslash Z=\{\Phi \rightarrow \Phi, \text { loại } \\
& \Phi \rightarrow \Phi, \text { loại } \\
& \Phi \rightarrow \Phi, \text { loại } \\
& e \rightarrow g, \\
& g \rightarrow e, \\
& \Phi \rightarrow \Phi, \text { loại }
\end{aligned}
$$

Vậy $\alpha=\left(U_{\alpha^{\prime}}, F_{\alpha}\right)$ vơi $U_{\alpha}=e g$, và $F_{\alpha}=\{e \rightarrow g, g \rightarrow e\}$. Lược đồ $\alpha$ dễ nhận thấy là chí có hai khoá là e và $g$, tức là $\mathcal{K}=\{e, g\}$. Và ta có: $\mathcal{K}_{\omega}=I_{\omega} \oplus \mathcal{K}_{\alpha}=h i \oplus\{e, g\}=\{e h i, g h i\}$. Như vậy lược đồ $\omega$ đã cho có hai khoá $\{e h i\}$ và $\{g h i\}$. 


\section{THIẾT KẾ VÀ CÀI ĐặT MODULE DABASCHE}

Phần này trình bầy phương pháp thiết kế và cài đặt module DABASCHE nhằm thể hiện các khái niệm lý thuyết đã trình bày trong các phần trước, đồng thời cung cấp một công cụ hũu hiệu cho những người thiết kế cơ sớ dữ liệu.

3.1. Chức năng chính của module

1.1. Chức năng khai báo một lược dồ quan hệ.

1.1.1. Cập nhậ̀t các thuộc tính của lược đồ.

1.1.2. Cập nhật các phụ thuộc hàm cưa lược đồ.

1.2. Chức năng xác định bao đóng của một tập thuộc tính.

Cho phép người sử dụng tìm bao đóng của một tập thuộc tính nào đó trong lược đồ đã khai báo.

1.3. Chức năng xáx định một khoá của lược đồ xuất phát từ một siêu khoá.

Chức năng này thể hiện thuật toán tìm khoá cơ bản dựa vào ý tưởng xuất phát từ một siêu khoá.

1.4. Chức năng thực hiện phép biến đổi lược đồ quan hệ theo một tập thuộc tỉnh cho trước: Đây là một chức năng thuận tiện phục vụ cho việc biến đổi linh hoạt lược đồ đã cho theo một tập thuộc tính nào đó.

1.5. Chức năng thực hiện phép biến đổi lược đồ quan hệ.

Chức năng này thể hiện lý thuyết về phép biến đổi lược đồ quan hệ đã trình bày trong phần II.

3.2. Cấu trúc dữ liệu

- Các đối tượng dữ liệu được chương trình xử lý là các thuộc tính, các phụ thuộc hàm, do đó tương úng có hai dạng cấu trúc dữ liệu sau đây:

2.1. Cấu trúc dữ liệu lưu các thuộc tính.

Để lưu các thuộc tính chúng ta xây dựng một "cây tìm kiểm" có dạng sau:

TreeU $={ }^{\wedge}$ Tree1;

Tree1 $=$ Record

Name : String[30]; \{ tên thuộc tính \}

B : Byte ; \{mã thuộc tính \}

Ord : Byte; \{ sõ thứ tự của thuộc tính \}

Left, Right : TreeU; \{con tró tró đến nút của cây \}

End;

2.2. Cấu trúc dữ liệu lưu các phụ thuộc hàm.

Cấu trúc danh sách liên kết kép để lưu các phụ thuộc hàm được xây dựng như sau: 
SetByte $=$ Set of byte;

Node $={ }^{\wedge}$ Node1;

Node1 $=$ Record

$$
\begin{aligned}
& \text { L,R : SetByte; }\{\text { lưu vế trái, vế phải của phụ thuộc hàm \} } \\
& \text { Pred, next : Node; }
\end{aligned}
$$

2.3. Một số đối tượng đặc biệt.

Vận dụng phương pháp lập trình hướng đối tượng, bộ chương trình đã xây dựng một số object phục vụ việc cập nhật các thuộc tính, các phụ thuộc hàm, cũng như tiến hành các xứ lý khác rất hiệu quá và thuận tiện.

Sau đây là một đối tượng cơ bản App2 hậu bối từ kiểu App1 đã xây dựng tṛong chương trinh:

$$
\text { App2 }=\text { Object }(\text { App1 })
$$

$\begin{array}{ll}\text { Constructor } & \text { Init; } \\ \text { Procedure } & \text { HandleEvent(Var Event: TEvent);Virtual; } \\ \text { Procedure } & \text { InitMenuBar;Virtual; } \\ \text { Procedure } & \text { UpdateA(Goc: TreeU; Attr: String[30]; } \\ & \text { Var Tap: SetByte; Them_Bot: Byte); } \\ \text { Procedure } & \text { UpdateFD; } \\ \text { Procedure } & \text { UpdateX(Var Tap: SetBNyte); } \\ \text { Procedure } & \text { ViewFD(GocUa: TreeU; DauList: Node; } \\ & \text { NameFile: String[8]; Var OFd, NFd: Byte; } \\ & \text { Var WinCount: Integer); } \\ \text { Procedure } & \text { ViewSFD(GocUa: TreeU; DList: NodeG; } \\ & \text { NameFile: String[8]; Tit: String; } \\ & \text { Var OFd, NFd: Byte; } \\ & \text { Var WinCount: Integer); } \\ \text { Procedure } & \text { View1Set(GocUa: TreeU; Tap: SetByte; } \\ & \text { NameFile: String[8]; Tit1, Tit2: String; } \\ & \text { Var OFd, NFd: Byte; } \\ & \text { Var WinCount: Integer); } \\ & \text { ViewTran(GocUa: TreeU; Tap: SetByte;Dau: NodeG; } \\ \text { Procedure } & \text { NameFile: String[8]; Tit1, Tit2, Tit3: String; } \\ & \text { Var WinCount: Integer); } \\ & \text { InitStatusLine; Virtual; }\end{array}$


End;

Các phương thức đầu Update: cập nhật các thuộc tính, các phụ thuộc hàm.

Các phương thức đầu View: hiễn thị các kết quả xử lý.

\section{KÊTT LUẬN}

Phương pháp xử dụng phép biến đổi các lược đồ quan hệ để xác định khoá như đã giới thiệu trong các phần nêu trên là một phương pháp hiệu quá để xác định khoá cưa lược đồ. Và module $\mathrm{DaBasche}$ là một bộ chương trình cài đặt thuận tiệc cho người thiết kế cơ sở dữ liệu.

\section{TÀI LIỆU THAM KHẢo}

1. Amstrong W.W., Dependency structures of database relationships (Information processing 74), North Holland Pub. Co. Amsterdam 1974.

2. Berri C., Berstein P.A., Computational problems related to the design of normal form relational schemes, ACM Translations on data base systems, v. 4 n. 11980.

3. Codd E.F., A Relational Model of Data for Large Shared Data Banks CACM 13:6 June 1970, 377-387.

4. Demetrovics J., Nguyen Xuan Huy, Closed sets and translations of relation schemes, Computer math. applic. v. 21, n. 1 1991, 13-23.

- 5. Horowitz E., Sahni S., Fundamentals of computer algorithms, Computer science press INC, Computer software engineering series, 1978.

6. Lê Văn Bào, Nguyễn Xuân Huy, Hồ Thuần, Demetrovics J., Lược đồ cân bằng và khoá của lược đồ quan hệ, Số đặc biệt về hai đề tài Nhà nước 48.05.08

7. Maier D. The theory of Relational Databases. Computer Science Press, Rockville, Md., 1983.

8. Niklaus w. Cấu trúc dữ liệu + Giải thuật $=$ Chương trỉnh, Nxb Thống kê, Hà nội, 1982 (Sách dịch).

9. Phạm Quang Trung, Một số vấn đề lý thuyết và lập trình cài đặt đám báo tính toàn vẹn của dữ liệu trong cơ sở dữ liệu quan hệ. (Luận văn Thạc sỹ tin học), Trường Đại học Bách khoa Hà nội, 1993.

10. Ullman J. D. Principles of Database Systems. 2nd. Computer Science Press. Potomac, Md, 1982. 